\title{
Application of Multi-level Recursive Method for Ship-Sway prediction
}

\author{
Shang-cheng Hu, Yong-gang Li, Xiang-ming Li, Sheng-ping Li and Yi Wang \\ China Satellite Maritime Tracking and Controlling Department, Jiangyin 214431,China
}

\begin{abstract}
The ship-sway is a non-stationary time series while ship sailing in the sea, when using the traditional orthogonal polynomial fitting,index filtering or Kalman filtering to predict ship-sway, the prediction error may be large, because the model parameters are fixed and cannot be adjusted in real time according to the measured data. Multi-level recursive method treats the dynamic system as a time-varying parameters of the system, and can be more in line with the objective reality of rocking the ship. After analyzing the characteristics of the ship-sway, the predict model established by multi-level recursive has been built and test results showed that this model can improve the prediction accuracy of the ship-sway data, and has some practical value in the prediction of the ship-sway.
\end{abstract}

Key words: Multi-level recursive,Ship-sway prediction,EViews,Stationary time series.

\section{Introduction}

In the tasks of maritime spacecraft tracking and control, the prediction of the measure base directly influences the data accuracy of radar while tracking the target in the way of following number-leading calculated by computer. At present, the ship-sway is the most important factor in the measure base, and the common ship-sway prediction methods include statistics-predict, Kalman filter-predict, orthogonal polynomial fitting-predict and maximum likelihood estimation-predict ${ }^{[1]}$. In reality application, some weakness of these methods is found, just like needing too much prior condition, too difficult to build the accurate ship-sway model, and too big predict deviation etc. Using multi-level recursive method, we can treat the ship-sway data as a random dynamic time varying system, further, separate the system predict to two steps, predict the dynamic time varying parameters and system status based on ahead parameters. By this way, the dynamic parameters will update real-time while the ship-sway make a sudden change, and the system prediction can have a good adaptability.

\section{Principle and Procedure of Multi-level Recursive}

\subsection{Principle of Multi-level Recursive}

The Multi-level Recursive treats a dynamic system as a stationary time series composed by one or more dimensional. By analysis the external characteristic of the system, establish the connection between inputs and outputs. Under the general circumstance, all systems can be simplified to one model includes one or more one-dimensional model [2]. Here, we suppose the input of a stationary time series is: 
$y(0), y(1), y(2) \cdots \cdots y(n)$

Inside the above expression, $y(k)$ is the observed value in the time of $k$, so the one-step prediction is:

$$
\begin{gathered}
\hat{y}(k+1 \mid k)=a_{1}(k) y(k-1)+\cdots \cdots+a_{n}(k) y(k-n)+ \\
b_{0}(k) u(k)+b_{1}(k) u(k-1)+\cdots \cdots+b_{m}(k) u(k-m)+e_{k}
\end{gathered}
$$

Inside the above expression, $u(k)$ is weight coefficient,

$$
\begin{aligned}
& a_{1}(k) \cdots \cdots a_{n}(k), b_{0}(k) \cdots \cdots b_{m}(k) \quad \text { are } \\
& \theta(k)=\left(a_{1}(k) \cdots \cdots a_{n}(k), b_{0}(k) \cdots \cdots b_{m}(k)\right)^{T}
\end{aligned}
$$

dynamic time varying parameters, $n, m$

And then $\{\theta(k)\}$ can be treated as a new expression for $\{\theta(k)\}$ : stationary time series, just like predict $y(k+1 \mid k)$ above, we can have the second

$$
\hat{\theta}(k+1 \mid k)=c_{0}(k) \theta(k)+c_{1}(k) \theta(k-1)+\cdots \cdots+c_{r}(k) \theta(k-r)
$$

Inside the above expression, $c_{0}, c_{1} \cdots \cdots c_{r}$ are dynamic time varying parameters, If the parameters are time varying, we can establish third, fourth or more further expression ${ }^{[3]}$, else the whole expressions make up the predict progress.

\subsection{Parameters Update}

According to the expression (1) (2) and reference documentation [4], the common expression of a predict progress is:

$$
y(k)=f\left(y_{k-1}, U_{k}, \theta, k\right)+v(k)
$$

In the real-time prediction of the analyses the ship-sway data series. So, the ship-sway by the multi-level recursive method, the factor $U_{k}$ can be ignored, only estimate of parameters $\theta$ is:

$$
\hat{\theta}(k)=\hat{\theta}(k-1)+\frac{\delta}{\|\varphi(k)\|^{2}} \varphi(k)\left[y(k)-\varphi^{T}(k) \hat{\theta}(k-1)\right]
$$

Inside the above expression, $\varphi(k)=(y(k-1), y(k-2) \cdots \cdots y(k-n))^{T}$, $\hat{\theta}(k)=\left(a_{1}(k) \cdots \cdots a_{n}(k)\right)^{T} \quad$ are estimated values, $\delta$ is adjust factor.

According to reference documentation [5], generally speaking, the different initial values of the parameters will not influence 
the predict results. But in real application, the most suitable initial values can shorten the predict time. So, least square method is used to calculate the initial values of the parameters. With the initial values and the expression (5), the parameters series can be estimated and updated:

$$
\hat{\theta}(1) 、 \hat{\theta}(2) 、 \hat{\theta}(3) \cdots \cdots \hat{\theta}(N)
$$

Based on the known series $\hat{\theta}$, we can predict $\theta(N+l)$ by methods include $\mathrm{AR}$ model, circle variable model, or mean value approximation model. Combined with

$$
\operatorname{FPE}(k)=\left(1+\frac{k+1}{n}\right) \times\left(1-\frac{k+1}{n}\right)^{-1} \delta_{e}^{2}
$$

Inside the above expression, $k$ is number of the model independent parameters, $n$ is the number of the samples, $\delta_{e}{ }^{2}$ is the error mean square deviation of the model.

\section{Multi-level Recursive Model for Ship-sway}

Using the method from reference documentation [9], we can simulate 1000 points ship-sway samples, after difference of first order, the new series is a stationary time series, and multi-level recursive model is built for predicting it. Here Eviews soft is expressions (1) (4), the $l$ steps prediction of $y$ can be calculated.

\subsection{Points of Multi-level Recursive Method}

According to reference documentations [6][7][8], whatever the model points selected, the predict precision will satisfy requirement by parameters stochastic makeup, But just like the model parameters initial values, the most suitable model points can shorten the predict time. So, Final Prediction Error criterion is used to define the most suitable model points:

used to get the model points and parameters initial values. Select Final Prediction Error criterion to have a unit-root test for the series and set the biggest lag order is $21^{[10]}$, the result shows figure 1 .

Though the orders D(-1)、D(-14)、D(-15) have no conspicuousness, considering the randomness, the model points is defined 16 . Using least squares method to estimate initial values of the parameters, the result shows figure 2 . 


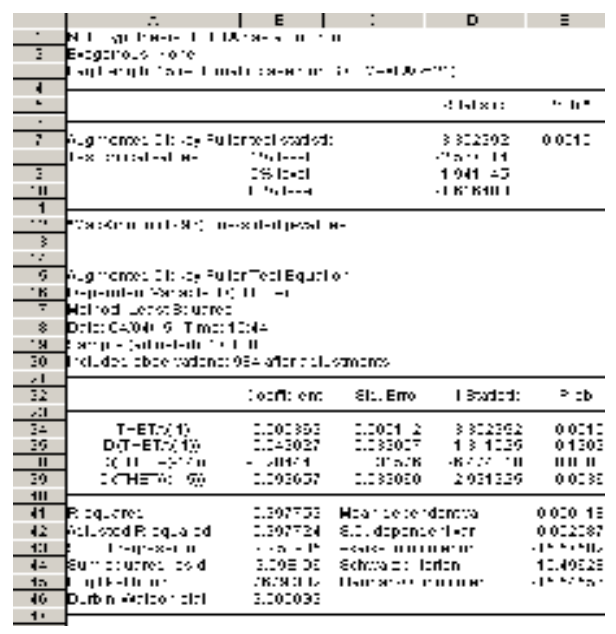

Fig1. Unit-Root Test Result

Thus, the initial values of the parameters $a(0) \sim a(16)$ are defined:

THETA $=0.00415521684124+[\mathrm{AR}(1)=1$. $04133890593, \mathrm{AR}(2)=0.432262930578, \mathrm{AR}$ (3) $=-0.22614528621, \mathrm{AR}(4)=-0.019044301$ $7317, \mathrm{AR}(5)=-0.0145654379696, \mathrm{AR}(6)=-0$. $37703029319, \operatorname{AR}(7)=0.0203289046708, \mathrm{~A}$

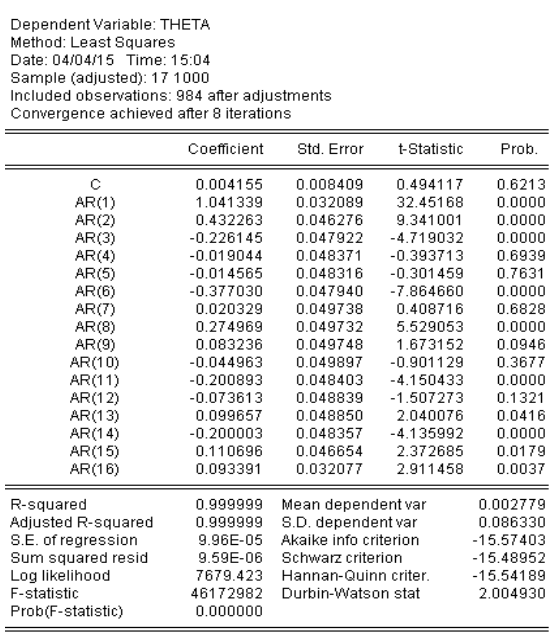

Fig2.Based on least squares method for estimating initial time-varying parameters

$\mathrm{R}(8)=0.274968931422, \mathrm{AR}(9)=0.08323627$ $95316, \operatorname{AR}(10)=-0.0449634962796, \operatorname{AR}(11)$ $=-0.200892928773, \mathrm{AR}(12)=-0.073613294$ $9649, \operatorname{AR}(13)=0.0996569990796, A R(14)=-$ $0.200003294775, \operatorname{AR}(15)=0.110696302707$ ,AR(16) $=0.0933908674749]$ 。

And meanwhile, the predict model expression is:

$$
\hat{y}(k+1 \mid k)=a_{16}(k) y(k-1)+\cdots \cdots+a_{1}(k) y(k-16)+a_{0}(k)
$$

According to expression (5), estimate and update parameters $a_{i}$ expressions are:

$$
\left\{\begin{array}{c}
\hat{a}_{0}(k)=\hat{a}_{0}(k-1) \\
\hat{a}_{1}(k)=\hat{a}_{1}(k-1)+\frac{1}{R(k)} y(k-16) V(k) \\
\cdots \\
\hat{a}_{16}(k)=\hat{a}_{16}(k-1)+\frac{1}{R(k)} y(k-1) V(k)
\end{array}\right.
$$

Above expressions:

$R(k)=\sum_{i=1}^{n} y^{2}(k-i) \quad 、 \quad V(k)=y(k)-\sum_{i=1}^{n} \hat{a}_{i}(k-i) y(k-i)$

\section{Predict Steps:}

Step1: According to the expressions (8) and the initial values of $a(0) \sim a(16)$, using the before 30 points samples, each parameter will form a series;

Step2: For each parameter series, establish a AR(3) model, and the one step prediction of the parameter can be get; 
Step3: With the predicted result of the parameters, the progress for predicting the ship-sway has been shown by the expression (7);
Step4: Compute the deviation between the samples and the prediction with the same time,figure them and the result shows figure 3.

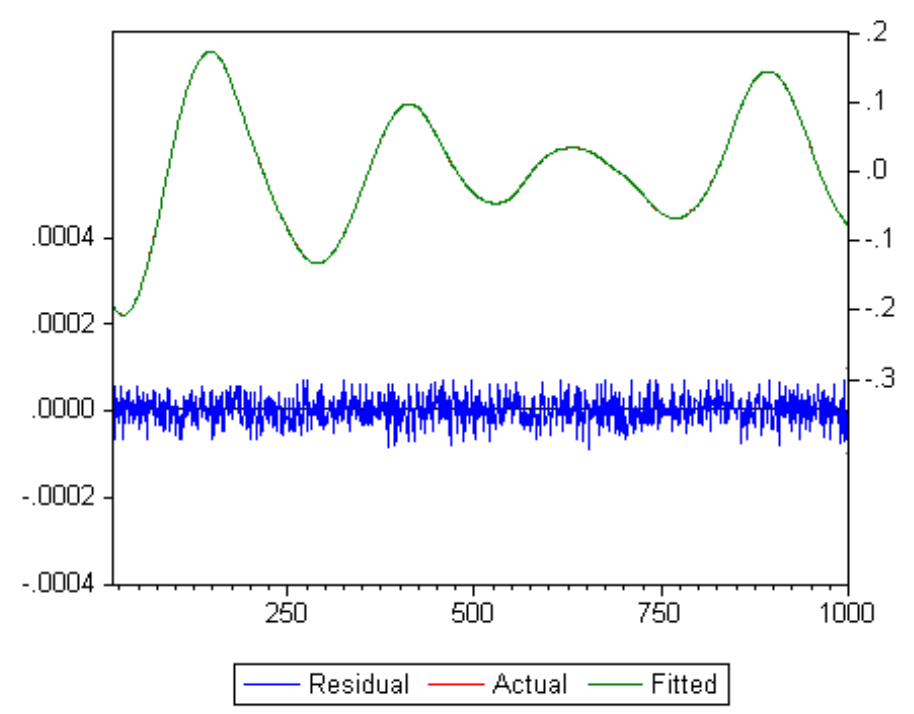

Fig3.Dynamic curve fitting step prediction and deviation results

Compute the maximum,mean and the results from reference documentation variance of the deviation, and compare with $\quad[10]$, the result shows table1.

Table1 Forecast accuracy deviation comparison

\begin{tabular}{c|c|c|c}
\hline Method/Deviation & Maximum/Arcseconds & Mean/Arcseconds & Variance/Arcseconds \\
\hline Index Filter & 13.899 & 3.753 & 5.512 \\
Time series & 6.376 & 1.662 & 2.443 \\
Multi-level Recursive & 4.352 & 1.355 & 2.459 \\
\hline
\end{tabular}

For verify when varying parameters dynamically adjusting benefits, when the ship rolling simulation data, select the data points between 400-500 increases a trend method as described above and spreadsheet modeling, comparative prediction deviation maximum, mean and variance, the results item (endpoint regression), using the same shown in table 2 .

Table2 Forecast accuracy deviation transition sequence comparison

\begin{tabular}{c|c|c|c}
\hline Method/Deviation & Maximum/Arcseconds & Mean/Arcseconds & Variance/Arcseconds \\
\hline Index Filter & 24.970 & 6.501 & 10.111 \\
Time series & 19.017 & 4.907 & 5.337 \\
Multi-level Recursive & 12.549 & 2.014 & 3.918 \\
\hline
\end{tabular}

From the spreadsheet results, when the status of the observed series change suddenly, the multi-level recursive method advantage is more obvious, with a faster adaptability and higher prediction accuracy。

\section{Conclusion}

Compared with traditional fixed parameter prediction method, multi-level recursive method forecast by layer by layer when varying parameters, dynamic adjustment of the estimated parameters in 
the prediction process, the study can be closer to the actual situation, a more sensitive and state recognition ability to adjust the prediction accuracy has also been greatly improved, with high practical value and popularization meaning.

\section{References}

[1]Zhao Xi-ren.Status of Ship-Modeling Sport Very Short-term Forecasts.Ship Engineering,J.

2002,3

[2] Wang Yun-ge. Multi-level Recursive Forecasting Method and Its Improvement,Journal of Hebei Normal University,J.1998,22(4)

[3] Han Zhi-gang. The Progress of Theory and Application of Multi-level Recursive Method,

Control and Decision,J.2001,16(2)

[4] Han Zhi-gang. A New Method of Dynamic Prediction System,Acta Automatica Sinica,J.

1983,3

[5] Han Zhi-gang. Parameter Estimation Initial for Multi-level Recursive Method, Control and Decision,J.1986,1(1)

[6] Luo Qin-bin. The Application of Multi-progressive Method to weather Forecast,Harbin University Institute,J. 2003,10

[7] Wang Fu. Research and Design for Energy Management Information System Based on Multi-level Recursive and Grey Prediction Theory, Kunming University of Science and Technology,J. 2010,3

[8] Chen Yu-xiang. Prediction and Applications,Machinery Industry
Press,BeiJing,1985, pp.98-135

[9] Liu Peng. Research on MATLAB based ocean wave and ship roll simulation models, ShanDong Science,J.2012,25(6)

[10] Zhang Zhong-hua. Boat Pose Data Processing Method for Space-tracking ship,National Defend Industry Press,BeiJing,2009, pp.219-293 